\section{§9. Induced Charge of Spherical Dust Particle on Plasma-Facing Wall in Non-uniform Electric Field}

Tomita, Y.,

Smirnov, R. (UCSD),

Zhu, S. (ASIPP)

In order to obtain the induced charge of the spherical dust particle on the plasma-facing wall, the bipolar coordinates are introduced and the local electrostatic potential was analyzed ${ }^{1)}$.

The electric field of the normal direction to the spherical dust surface gives the charge density $\sigma_{S}(\alpha)$ at the spherical dust surface $\left(\beta=\beta_{0}=1 / 2\right)$ :

$$
\begin{aligned}
\sigma_{S}(\alpha)= & \left.\varepsilon_{0} E_{n}\right|_{\beta=\beta_{0}=1 / 2}=\left.\varepsilon_{0} \frac{\alpha^{2}+\beta_{0}^{2}}{R_{d}} \frac{\partial \phi}{\partial \beta}\right|_{\beta=\beta_{0}=1 / 2} \\
= & -\varepsilon_{0} \frac{\left(\alpha^{2}+\beta_{0}^{2}\right)^{3 / 2}}{R_{d}}\left\{h_{1} R_{d} I_{c 20}(\alpha)\right. \\
& +\frac{h_{2} R_{d}^{2}}{3}\left[\frac{I_{10}(\alpha)}{\beta_{0}^{2}}+\frac{I_{c 20}(\alpha)}{\beta_{0}}+I_{c 30}(\alpha)\right] \\
& +\frac{h_{3} R_{d}^{3}}{5}\left[\frac{2 I_{10}(\alpha)}{\beta_{0}^{3}}+\frac{I_{c 20}(\alpha)}{\beta_{0}^{2}}+\frac{I_{20}(\alpha)}{\beta_{0}^{2}}\right. \\
& \left.+\frac{I_{c 30}(\alpha)}{\beta_{0}}+\frac{I_{c 40}(\alpha)}{3}\right] \\
& +\frac{h_{4} R_{d}^{4}}{7}\left[\frac{3 I_{10}(\alpha)}{\beta_{0}^{4}}+\frac{I_{c 20}(\alpha)}{\beta_{0}^{3}}+\frac{2 I_{20}(\alpha)}{\beta_{0}^{3}}\right. \\
+ & \left.\frac{I_{c 30}(\alpha)}{\beta_{0}^{2}}+\frac{2 I_{30}(\alpha)}{5 \beta_{0}^{2}}+\frac{2 I_{c 40}(\alpha)}{5 \beta_{0}}+\frac{I_{c 50}(\alpha)}{15}\right](1)
\end{aligned}
$$

where $I_{n 0}(\alpha) \equiv \int_{0}^{\infty} d \lambda \lambda^{n} e^{-\beta_{0} \lambda} J_{0}(\lambda \alpha)$ and

$I_{c n 0}(\alpha) \equiv \int_{0}^{\infty} d \lambda \lambda^{n} \frac{J_{0}(\lambda \alpha)}{\sinh \left(\beta_{0} \lambda\right)}$.

The induced charge $Q_{\text {din }}$ on the conducting spherical dust is obtained

$$
\begin{aligned}
& Q_{\text {din }}=\int_{S_{d}} \sigma_{s} d S \\
& =-\left.2 \pi_{\varepsilon_{0}} R_{d}^{2} \int_{0}^{\infty} \frac{\alpha}{\left(\alpha^{2}+\beta^{2}\right)^{2}} \frac{\partial}{\partial \beta}\left(\phi_{e x}+\phi_{i n}\right)\right|_{\beta=\beta_{0}=1 / 2} d \alpha \\
& =-2 \pi_{\varepsilon_{0}} R_{d}^{2}\left(d_{1} h_{1}+d_{2} h_{2} R_{d}+d_{3} h_{3} R_{d}^{2}+\cdots\right)
\end{aligned}
$$

where $\sigma_{s}$ and $S_{d}$ are the surface charge density (eq.(14)) and the dust surface area, respectively. In case of $k_{\max }=4$, the numerical coefficients $d_{k}$ 's are

$$
d_{1}=I_{q c 2} \cdot d_{2}=\frac{1}{3}\left(\frac{I_{q 1}}{\beta_{0}^{2}}+\frac{I_{q c 2}}{\beta_{0}}+I_{q c 3}\right)
$$

$$
\begin{gathered}
d_{3}=\frac{1}{15}\left[\frac{6 I_{q 1}}{\beta_{0}^{3}}+\frac{6\left(I_{q 2}+I_{q c 2}\right)}{\beta_{0}^{2}}+\frac{3 I_{q c 3}}{\beta_{0}}+I_{c 4}\right] \\
d_{4}=\frac{1}{105}\left[\frac{45 I_{q 1}}{\beta_{0}^{4}}+\frac{15\left(2 I_{q 2}+I_{q c 2}\right)}{\beta_{0}^{3}}\right. \\
\left.+\frac{3\left(2 I_{q 3}+5 I_{q c 3}\right)}{\beta_{0}^{2}}+\frac{6 I_{q c 4}}{\beta_{0}}+I_{q c 5}\right]
\end{gathered}
$$

where $I_{q n} \equiv \int_{0}^{\infty} d \alpha \frac{\alpha I_{n 0}(\alpha)}{\sqrt{\alpha^{2}+\beta_{0}^{2}}}$

$$
\begin{aligned}
& =\int_{0}^{\infty} d \alpha \frac{\alpha}{\sqrt{\alpha^{2}+\beta_{0}^{2}}} \int_{0}^{\infty} d \lambda \lambda^{n} e^{-\beta_{0} \lambda} J_{0}(\lambda \alpha) \\
& =\int_{0}^{\infty} d \lambda \lambda^{n-1} e^{-2 \beta_{0} \lambda}=\Gamma(n) /\left(2 \beta_{0}\right)^{n} \\
\text { and } \quad I_{q c n} & \equiv \int_{0}^{\infty} d \alpha \frac{\alpha I_{c n 0}(\alpha)}{\sqrt{\alpha^{2}+\beta_{0}^{2}}} \\
& =\int_{0}^{\infty} d \alpha \frac{\alpha}{\sqrt{\alpha^{2}+\beta_{0}^{2}}} \int_{0}^{\infty} d \lambda \lambda^{n} \frac{J_{0}(\lambda \alpha)}{\sinh \left(\beta_{0} \lambda\right)} \\
& =\int_{0}^{\infty} d \lambda \frac{\lambda^{n-1} e^{-\beta_{0} \lambda}}{\sinh \left(\beta_{0} \lambda\right)}=2 \Gamma(n) \varsigma(n) /\left(2 \beta_{0}\right)^{n}
\end{aligned}
$$

Here $\Gamma(n)$ and $\zeta(n)$ are the Gamma and Rieman's Zeta functions, respectively. The first term of the RHS corresponds to the charge in the uniform electric field.

The induced charge of the conducting spherical dust particle on the conducting wall in the non-uniform electric field was calculated theoretically as well as the local electrostatic potential. The non-uniform electrostatic potential is approximated by the polynomial of the normal coordinate toward the wall. These results can be compared to the results from the particle computer simulation [6], where the total charge includes both of effects of induced charges and plasma shielding. This theoretical analysis is useful to understand the dust behavior in the boundary plasma.

\section{Reference}

1) Tomita, Y., Smirnov R., and Zhu S., Plasma Science and Technology, 8 (2006) 122-124. 